\title{
Driver Fatigue Recognition using Skin Color Modeling
}

\author{
Md. Mehedi Hasan \\ Department of ECE \\ Khulna University of \\ Engineering \& Technology \\ Khulna-9203, Bangladesh
}

\author{
Md. Foisal Hossain \\ Department of ECE \\ Khulna University of \\ Engineering \& Technology \\ Khulna-9203, Bangladesh \\ Prajoy Podder \\ Department of ECE \\ Khulna University of \\ Engineering \& Technology \\ Khulna-9203, Bangladesh
}

\author{
Jag Mohan Thakur \\ Department of ECE \\ Khulna University of \\ Engineering \& Technology \\ Khulna-9203, Bangladesh
}

\begin{abstract}
In Driver's fatigue is a major safety concern in transportation system, because driver drowsiness and distraction have been casual factor for the large number of road accident. Fatigue reduces the driver's perception level and decision making ability, which responsible for serious road accident. Around $22 \%-24 \%$ of car crash occurred by driver drowsiness. There is a way to reduce these accidents by monitoring drives fatigue and driving behaviors at the driving time by alerting the drivers, while the drivers are drowse. Face detection, eyes state measurement, lip detection, yawing detection, head tilting detection are the major visual facial symptoms for the driver fatigue detection. In this paper a modern assistive frame work has been introduced, which detected driver drowsiness based on visual features measurement. The goal of this paper has been monitored the driver driving behaviors, to detect the visual facial symptoms for safe driving in the road. Facial features symptoms have been monitored by two cameras. To detect driver distraction, the proposed algorithm has been experimented the facial fatigue expression, head tilting and lane departure. Experimental result of the proposed method has been compared with the existing methods. The experimental results show that, the proposed algorithm has good accuracy and reliable performance to reduce the road accident than the existing methods. The average accuracy of the proposed method is $92.44 \%$.
\end{abstract}

\section{Keywords}

Driver fatigue, Driver fatigue monitoring, Fatigue symptoms detection, Fatigue warning system, Lane exodus, Region localization, Skin color segmentation.

\section{INTRODUCTION}

Driver fatigue is one of the general problems in road accidents in the whole world. The common reasons for fatigue among car drivers these days are sleep disorders, deprivation, inattention and illness [1]. The National Highway Traffic Safety Administration (NHTSA) estimated that 56,000 of total crashes are caused by drowsy drivers due to which 1,550 fatalities and 40,000 injuries occur every year in U.S. According to American drivers $70 \%$ of total accidents occurred due to driving fatigued. National Transportation Safety Board (NTSB), in the case of trucking industry $57 \%$ of total fateful truck accidents occurred due to driver fatigue. This is the number one cause of heavy truck crashes [2]. It is estimated that approximately $25 \%$ of police reported crashes occur due to some form of driver inattention situations. When the driver is lost in thought, sleepy or distracted and other estimates of driver inattention range is as high as $35-50 \%$ [3]. According to a study by the Sleep Research Center (UK), $20 \%$ of accidents on monotonous roads occur due to driver drowsiness at the wheel. At present 20 to 50 million people suffer non-fatal injuries due to road accidents and over 1.3 million people die each year on the road. According to age group studies, it is showed that 18 to 20 year old drivers experienced four times of inattention related crashes in the comparison of older drivers [4]. In the year 2003, French statistics showed that fatigue are the major causes of accidents than alcohol or sleep deprivation. Studies also showed that collision due to drowsiness mainly occurred during late night or early morning hours. Shift workers, people with untreated sleep disorders and commercial vehicle operators have great risk for such collisions.

Fatigue is a physical or mental exhaustion that can be triggered by inadequate rest, improper diet, work and home stressors. During the last few years, many techniques have been proposed for driven fatigue recognition system. These techniques are classified into three categories [5]. Intrusive technique based on monetarily biomedical signals, nonintrusive technique based on visual features of driver's face image and vehicle based features based on driver driving performance behavior. Computer Vision Method based on non-intrusive technique are particularly effective because driver fatigue can be recognized by monitoring the facial features such as face, eyes state, yawning and head position. Face detection methods are classified into three categories [6]. Knowledge based method, Template matching method and Appearance based method. Knowledge based method describes the features of a face [7]. Face recognition is parameterized by using a function in template matching method. Several sub templates are used to give input image and the correlation values with the standard pattern are computed for the face contour. The existence of face is determined based on these correlation values [8]. Neural network based method [9] is used for learning the face and non-face region from the face and non-face images. In case of eyes state detection, Template matching method [10] is used to determined eyes condition. Contour information [11] is used to determine eyes open or not and structured images are used for this purpose. After obtaining the contour images of eye, Hough Transform is used to detect the eye condition. Eyes are also detected by Gray Scale image [12], [13]. The 
open condition [14] of mouth has been detected by contrast stretching on the basis of pixels cumulative distribution. It is performed on the lower half fraction of the image pixels. As a result, lips and other darker region become darker and skin region becomes brighter providing a clear separation boundary between foreground and background. Support Vector Machine (SVM) [15] is used to classify the mouth regions to detect yawning. The work presented in [16] suggested that fatigue can be identified considering the driver's facial pose. Head tilt can be detected by the driver's facial orientation and HAAR like features [17]. The lane detection for the driver's fatigue is based on the edge detection at the first generation. The work described in [18] depends on, a perceptual grouping at edge point into image to detect the lane markers. Hough Transform is used to detect the lines on the road [19]. A lane departure decision is made by using the distance between Hough origin and the lane line mid-point.

Color is an important feature for driver driving behavior symptoms detection. Human skin color segmentation is faster than other existing methods to detect the symptoms. Skin color mainly depends on chrominance plane [20] or HIS. The most used color spaces are RGB, HSV (Hue Saturation Value), HIS (Hue Intensity Saturation) [21], YCbCr [22], CYM (Cyan Yellow Magenta), LHS, UVW. The aim of this paper is to discuss drive's fatigue recognition based on two procedures. First one is driver visual driving behavior features and the second one is driver's driving performance. Generally, driver's drowsiness symptoms are eyes open or close, yawing and head tilting. Lane departure is detected by edge detection and Hough transforms process. The proposed method has been used two cameras. The driver's driving behavior has been monitored by one camera and the driving vehicle position in the road is measured by another camera. According to the proposed system, at first face has been detected from the driver image by skin color segmentation process. The $\mathrm{YCbCr}$ color space [22], [23] is used for driver skin color segmentation. $\mathrm{YCbCr}$ is color space, which has $\mathrm{Cr}$ and $\mathrm{Cb}$ components and $\mathrm{Cr}$ is bigger than $\mathrm{Cb}$. Eyes state, mouth condition and head tilting have been detected from face image after face detection. Hough transforms and edge detection technique has been used to detect the lines at the road for making departure decision.

The rest of the paper is organized as follows. The overview of proposed algorithm is described on section-2. Under this section, face detection, eyes state detection, Mouth detection, Yawning detection, Head tilting detection, Lane departure detection and Warning system are explained clearly. The experimental results and comparison is discussed in section-3. Finally, section-4 concludes the paper.

\section{OVERVIEW OF PROPOSED ALGORITHM}

The proposed system has two part, open part is for driver facial fatigue behavior symptoms detection and another one is for lane departure detection. Alarming system has been designed from the decision of two parts.

Fig. 1 describes the total evaluation process of the proposed systems. Driver fatigue symptoms eye state, yawing, head tilting has been detected by using camera 1 . The vehicle position in the road has been monitored by using camera 2 and alarmed if driver fatigue is found.

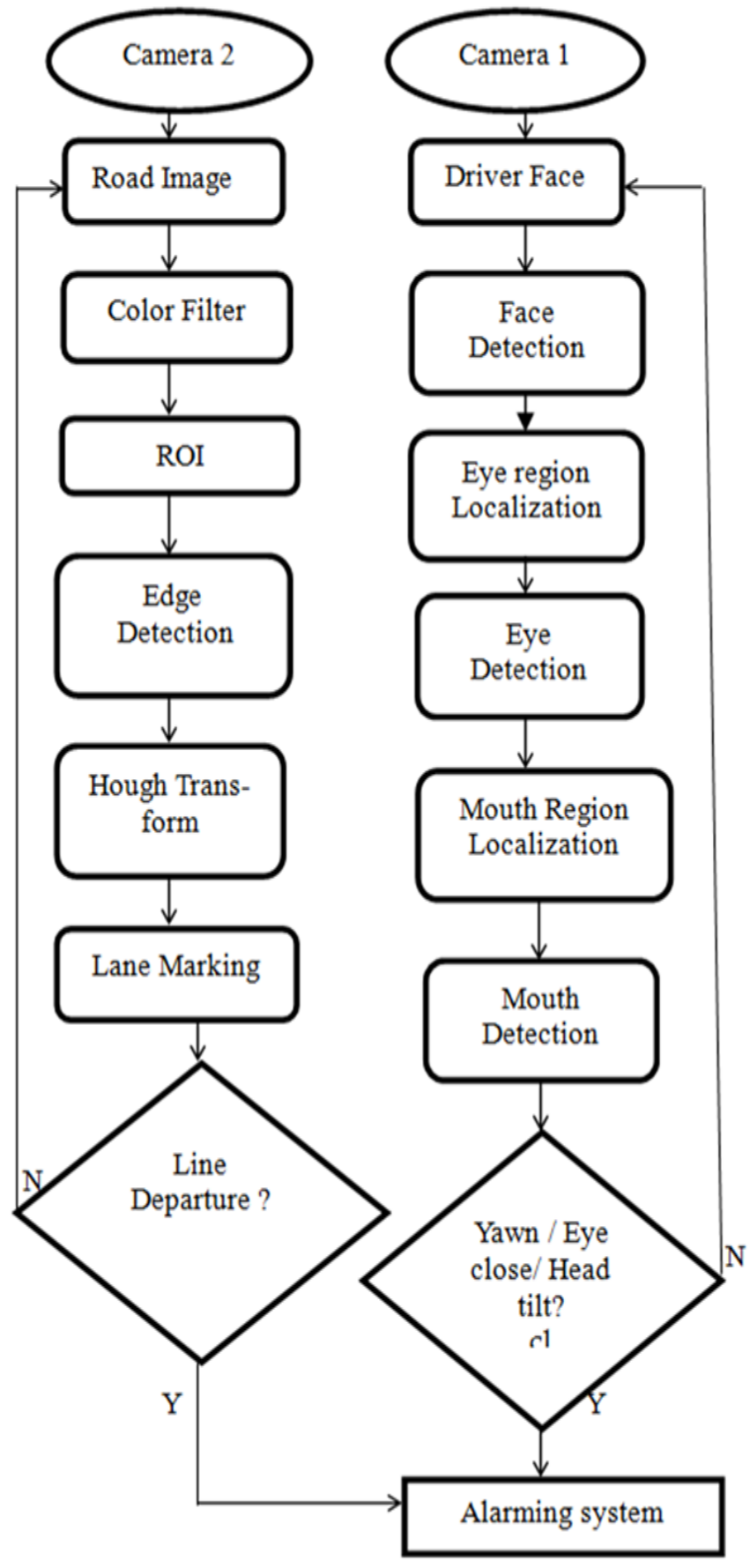

Fig.1: Block diagram of proposed method

\subsection{Face Detection}

Face detection is not a straight forward because it has lots of variation of image appearance, such as pose variations, Occlusion, image orientation, illuminating condition and facial expression. For driver face monitoring a camera has been fixed and it is also useful for the head tilt detection. Skin color segmentation process has been used for face detection, where $\mathrm{YCbCr}$ [23] is the main color space. The illumination variation problem [20] at images has been reduced by converting $\mathrm{RGB}$ to $\mathrm{YCbCr}$ and the following condition has been followed to face detection. 


$$
\left.\begin{array}{l}
80 \leq \mathrm{Cb} \leq 120 \text { and } \\
140 \leq \mathrm{Cr} \leq 193 \text { and } \\
60 \leq \mathrm{Y} \leq 255
\end{array}\right\}
$$

After skin color segmentation, the remaining small noise has been reduced by image erosion, image dilation through morphological technique. Binary image conversion helps image filling operator, which fills unwanted holes in face area. Exterior boundary points of the face region have been traced to detect the right, left, top and bottom side's points.
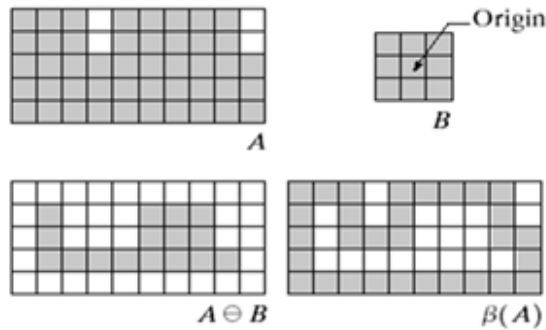

Fig.2: Exterior boundary extractions

Fig. 3 describes the result of face detection by the proposed method. Fig. 2(a) and fig. 2(b) represents different pose of face, fig. 2(c) signifies the detection of the multi face in the same image.
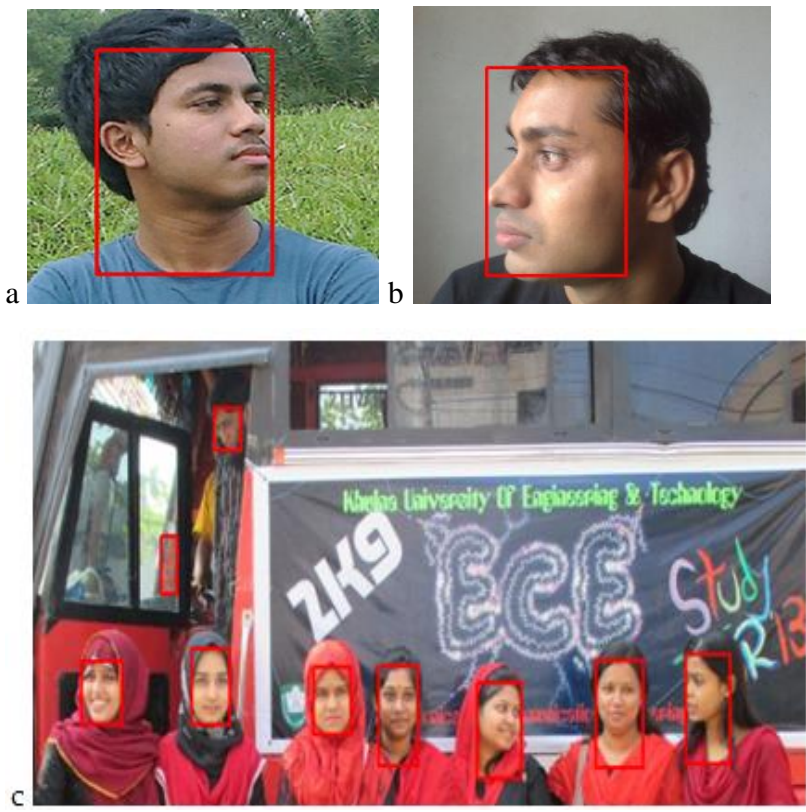

Fig.3: Results of face detection by proposed method

\subsection{Eye State Detection}

To recognize driver status the eyes state should be detected ahead. The eyes states can be classified into three types, full open, half open and close. Detected face image is used as input for the eye detection section. The region of interest for eyes has been localized from the upper part of the detected face by the following rules [24],

Eye_region_top=face_below+ $(.2 \times$ face_height $)$

Eye_region_below=face_top- $(.5 \times$ face_height $)$

Eye_region_right=face_right $+\left(.1 \times f a c e \_w i d t h\right)$

$$
\text { Eye_region_left=face_left-(.1×face_width) }
$$

After region of interest (ROI) localization, eye state has been detected by skin color segmentation process. Since YCbCr [22], [23] is the main color space for skin color segmentation. So, the following condition of the $\mathrm{Cb}, \mathrm{Cr}$ and $\mathrm{Y}$ has been followed for eye region skin segmentation.

Eye_region $=\left\{\begin{array}{c}1, \quad \text { if }(80 \leq C b \leq 117 \text { and } \\ 140 \leq C r \leq 193 \text { and } 40 \leq Y \leq 255 \\ 0, \text { otherwise }\end{array}\right\}$

\subsection{Mouth Detection}

Mouth features are important for determining driver fatigue. As yawing is one of the important states of the mouth that indicates driver fatigue. For yawn or gaping detection, at first mouth state has been detected that it is open or not. The proposed method has been localized the mouth region from the lower part of the detected face by following rules [24],

Mouth_region_top=face_below+ $(2.4 \times($ face_height/3.7) $)(7)$

$$
\begin{aligned}
& \text { Mouth_region_below=face_top- }(.1 \times \text { face_height }) \\
& \text { Mouth_region_right=face_right }+(.25 \times \text { face_width }) \\
& \text { Mouth_region_left=face_left- }(.15 \times \text { face_width })
\end{aligned}
$$

Where,

face_top: y coordinates of the top border of face region.

face_below: y coordinates of the below border of face region. face_left: $x$ coordinates of the left border of face region. face_right: $\mathrm{x}$ coordinates of the right border of face region. face_height: the height of face region.

face_width: the width of face region.

After mouth region localization, the proposed method is tranquil to detect mouth. The following condition of $\mathrm{Cb}, \mathrm{Cr}$ and $\mathrm{Y}$ has been used for the mouth region skin segmentation.

Mouth_region $=\left\{\begin{array}{c}1, \quad \text { if }(80 \leq C b \leq 116 \text { and } \\ 140 \leq C r \leq 193 \text { and } 60 \leq Y \leq 255 \\ 0, \text { otherwise }\end{array}\right\}$

\subsection{Yawn Detection}

Yawning occurred during period of increasing fatigue and brings a reversal or suspension process to fall asleep. Skin segmentation technique has been used for yawn detection because yawns consists of the typical sequence of respiratory, opening of jaw, closure of eyes, and contraction of facial muscles. After ROI localized from lower part of the detected face, it is easy to detect yawn from detected face image because yawning is the largest hole in the face image. At the time of yawn, the eyes area becomes narrow and large hole has been detected easily.

\subsection{Head Tilt Detection}

Head tilting is one of the significant symptoms of fatigue, which is rarely used. Head tilting found when driver feels extensive tiredness and driver's tends to release muscles pressure on their neck. As a result, head bends right or left side, considering driver is wearing seat belt. The amount of the tilt angle is calculated from the eye position by drawing straight line. The center coordinates of two eye has been used to draw the straight line by the following equation, 


$$
\frac{x-x 1}{x 1-x 2}=\frac{y-y 1}{y 1-y 2}
$$

Head tilt angle $(\Delta)$ has been determined by the following trigonometric rules,

$$
\begin{array}{cc}
\text { Tilt }_{\text {Angle }}=\tan ^{-1}\left(\frac{A B}{O B}\right) & \text { Radian } \\
\text { Tilt }_{\text {Angle }}=\text { Tilt }_{\text {Angle }} * \frac{180}{\pi} & \text { Degree }
\end{array}
$$
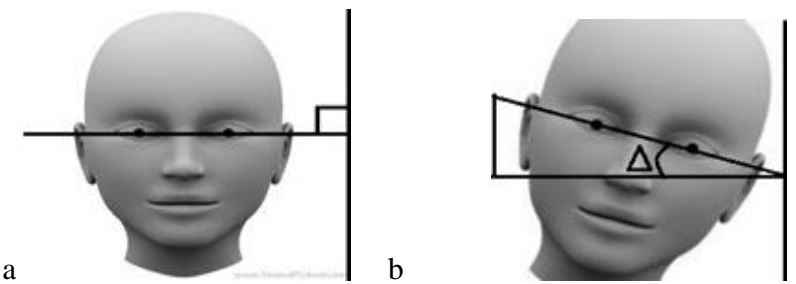

Fig.4: Head tilt angle calculation

\subsection{Lane Detection}

For safe driving, it is need to identify vehicle current position from the lane marker. When driver at a vehicle is in fatigue condition there is a large possibility for the vehicle to depart the lane marker. In this paper the proposed system has been fixed a camera to warn the driver's, at the time of vehicle depart from usual lane marker. There are some situations where Red and Green color pixels are dominant over white color pixels at lane marker. Color filter has been used to convert those red and green pixels to white pixels. Lane marker detection from the whole road image through edge detection technique or Hough transform technique is complex. So, Region of Interest (ROI) has been taken to avoid this complexity. After region localized, Hough transform is applied to find out the lane marker.

Fig. 4 shows head tilt angle calculation. Fig. 4(a) shows head normal condition and fig, 4(b) shows the head tilt condition.

Fig. 5 describes color filter output of road image, Fig. 5(a) shows original image, Fig. 5(b) represents red band image, Fig. 5(c) represents green band image, Fig. 5(d) represents blue band image
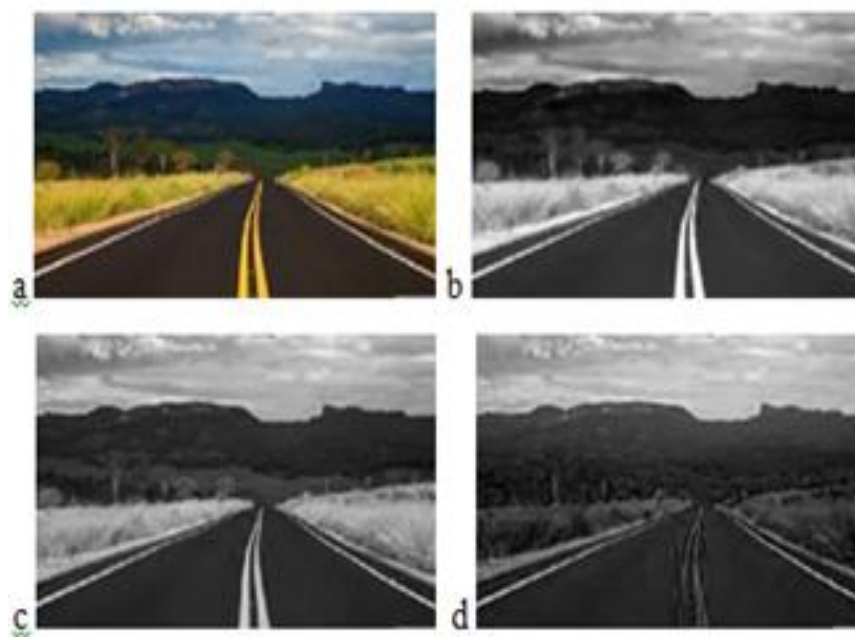

Fig.5: Color filter output of road image

\subsection{Warning System}

It is obvious to notify the driver if they are moving across the line.suppose driving vehicle at left side is the traffic rule.we designed our alert system through below algorithm:

IF( distance from focus point to left lane > distance from focus point to right lane )

\section{Start count time}

Else

$$
\begin{aligned}
& \text { Stop count time } \\
& \text { IF(time>thresold) } \\
& \text { Provide an alarm } \\
& \text { End of if else block }
\end{aligned}
$$

End of system

\section{EXPERIMENTAL RESULTS}

To demonstrate the performance of proposed algorithm, the presented algorithm has been implemented by using matrix laboratory software (MATLAB). The images are used as input to obtain from the Samsung company digital camera and the image size is $2592 \times 1944$ pixels.

Fig. 6 shows the result of face detection. Fig. 6(a) represents the input face image, fig, fig. 6(b) represents face boundary and fig. 6(c) represents detected face image.

Fig. 7 shows the result of open eye detection. Fig. 7(a) represents detected face image, fig. 7(b) represents dilate image and fig. 7(c) represents the detected open eye.

Fig. 8 shows the result of close eye detection. Fig. 8(a) represents detected face image, fig. 8(b) represents dilate image and fig. 8(c) represents the detected no eye.

Fig. 9 shows the result of mouth detection. Fig. 9(a) represents detected face image, fig. 9(b) represents connected component image and fig. 9(c) represents the detected mouth.

Fig. 10 shows the result of yawn detection. Fig. 10(a) represents detected face image, fig. 10(b) represents connected component image and fig. 10(c) represents the detected yawn.
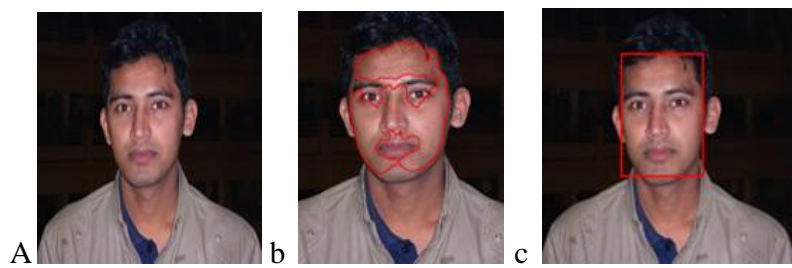

Fig.6: Results of face detection using the proposed method
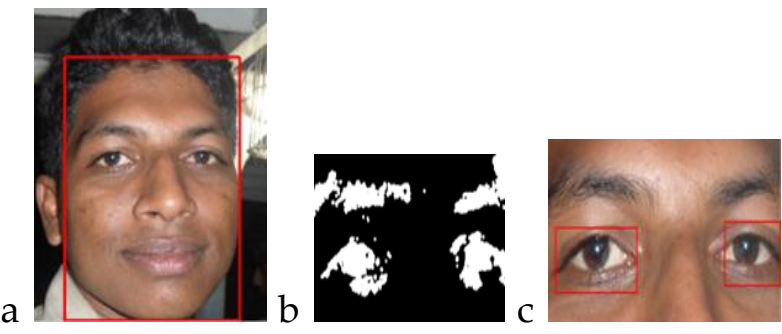

Fig.7: Results of open eye detection using the proposed method 
a
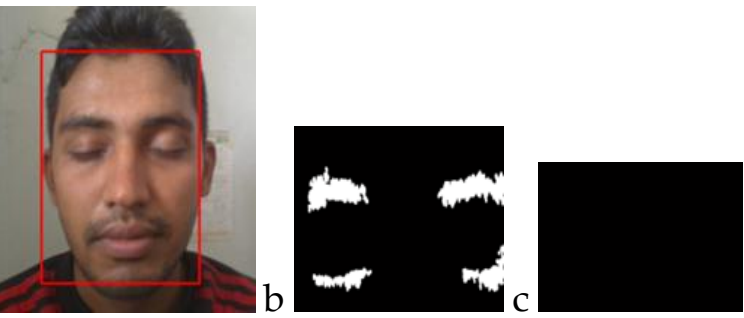

Fig.8: Results of close eye detection using the proposed method
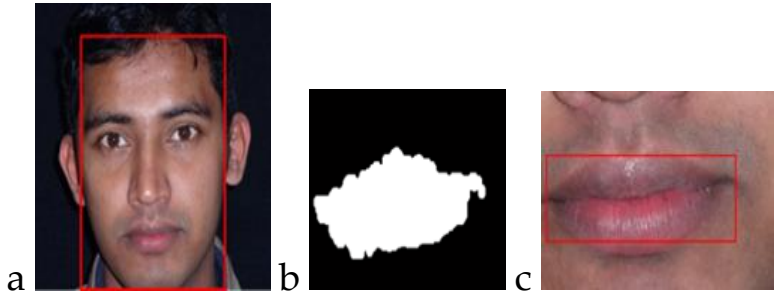

Fig.9: Results of mouth detection using the proposed method
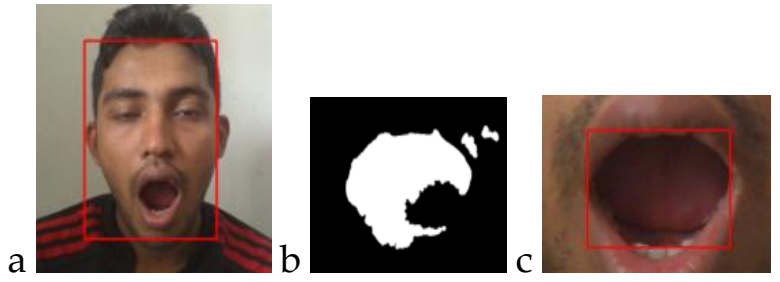

Fig.10: Results of yawing detection using the proposed method

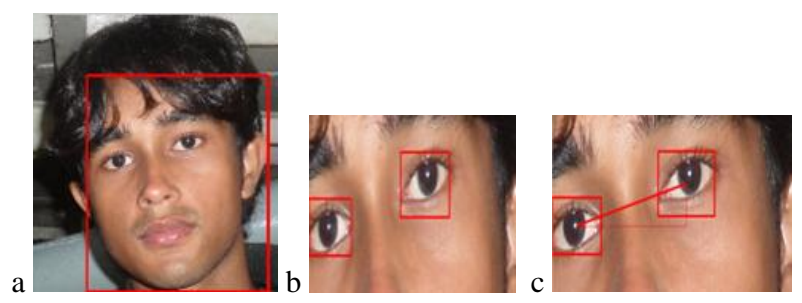

Fig.11: Results of head tilts detection using the proposed method
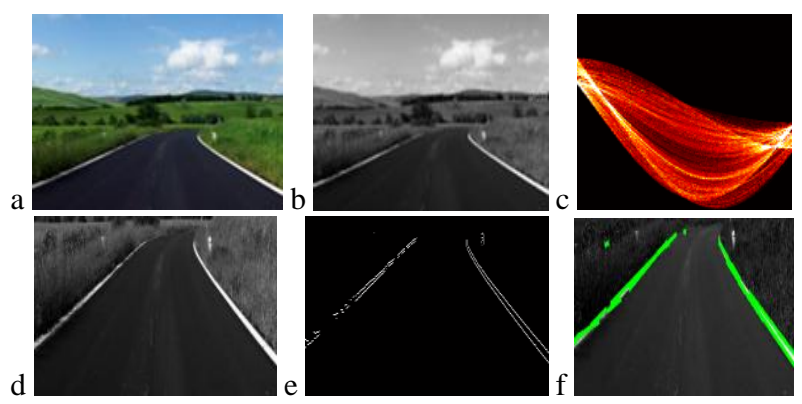

Fig.12: Results of lane departure detection using the proposed method
Fig. 11 shows the result of head tilt detection. Fig. 11(a) represents detected face image, fig. 11(b) represents eye detected image and fig. 11(c) represents the head tilt angle determination image. Fig. 12 shows the result of lane marker detection. Fig. 12(a) represents input road image, fig. 12(b) represents color filter output, fig. 12(c) represents road ROI, fig. 12(d) displays Hough matrix, fig. 12(e) represents edge detection image and fig. 12(f) represents the Hough transform $\&$ lane marking image.

The proposed algorithm has been experimented on 175 KUET students face image. All the face and road images have been given as input to the previous and proposed method [2]. Previous method is efficient for the close eye detection but it cannot detect lane marker. The faces in the images of the experimented people were different position, pose, facial expression, color condition and orientation. Table 1 show the outcome of the previous system. Table 2 shows the accuracy of the proposed system, where face detection is $98.9 \%$, eyes closer detection is $97.3 \%$, yawing detection is $94.7 \%$, head tilt detection is $84 \%$ and lane marker detection is $88 \%$. Table 3 shows the comparisons result between the previous method and proposed method. Investigates all the three tables, it is clear that the proposed method is better than the previous existing methods. So, it can be said that the proposed method is more efficient to detect the driver's fatigue accurately.

Table 1. Fatigue Detection by the Previous System

\begin{tabular}{|c|c|c|c|c|}
\hline Detection & $\begin{array}{c}\text { No Of } \\
\text { Image }\end{array}$ & $\begin{array}{c}\text { Perfect } \\
\text { Detection }\end{array}$ & $\begin{array}{c}\text { False } \\
\text { Detection }\end{array}$ & Efficiency \\
\hline Face & 150 & 149 & 01 & $99.3 \%$ \\
\hline Close Eye & 150 & 149 & 01 & $99.3 \%$ \\
\hline Yawing & 150 & 122 & 28 & $81.3 \%$ \\
\hline Head Tilt & 150 & 97 & 53 & $65 \%$ \\
\hline $\begin{array}{c}\text { Lane } \\
\text { Marker }\end{array}$ & 150 & 0 & 150 & $0 \%$ \\
\hline
\end{tabular}

Table 2. Fatigue Detection by the Proposed System

\begin{tabular}{|c|c|c|c|c|}
\hline Detection & $\begin{array}{c}\text { No Of } \\
\text { Image }\end{array}$ & $\begin{array}{c}\text { Perfect } \\
\text { Detection }\end{array}$ & $\begin{array}{c}\text { False } \\
\text { Detection }\end{array}$ & Efficiency \\
\hline Face & 150 & 148 & 02 & $98.9 \%$ \\
\hline Close Eye & 150 & 146 & 04 & $97.3 \%$ \\
\hline Yawing & 150 & 142 & 08 & $94.7 \%$ \\
\hline Head Tilt & 150 & 126 & 24 & $84 \%$ \\
\hline $\begin{array}{c}\text { Lane } \\
\text { Marker }\end{array}$ & 150 & 132 & 18 & $88 \%$ \\
\hline
\end{tabular}

Table 3. Comparison the Proposed method with previous method

\begin{tabular}{|c|c|c|}
\hline Detection & $\begin{array}{c}\text { Previous } \\
\text { Method }\end{array}$ & $\begin{array}{c}\text { Proposed } \\
\text { Method }\end{array}$ \\
\hline Face & $99.3 \%$ & $98.3 \%$ \\
\hline Close Eye & $99.3 \%$ & $97 \%$ \\
\hline Yawing & $81.3 \%$ & $94.9 \%$ \\
\hline Head Tilt & $65 \%$ & $84 \%$ \\
\hline Lane Marker & $0 \%$ & $88 \%$ \\
\hline
\end{tabular}

Fig. 13 shows the comparative chat between the previous and proposed methods. 


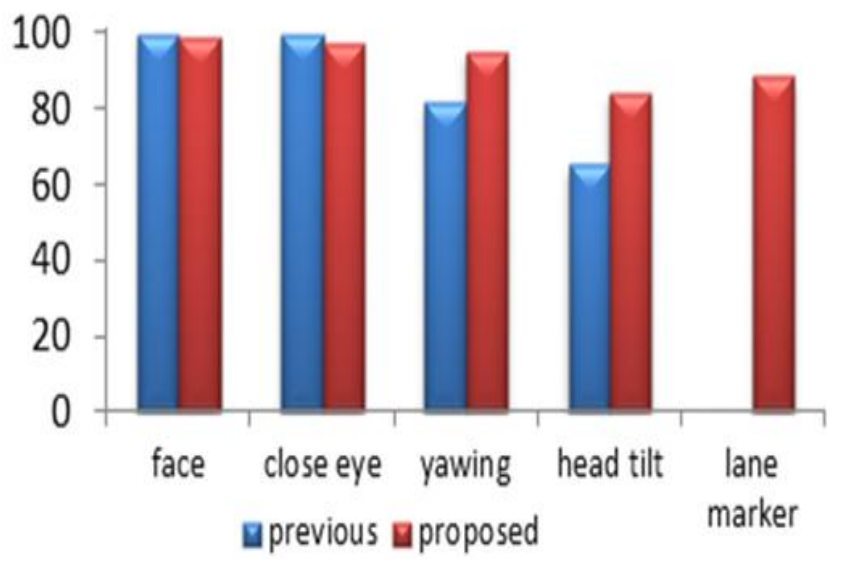

Fig.13: Comparative chat between previous and proposed method

\section{CONCLUSION}

In this paper an efficient method has been introduced, which consider most of the fatigue symptoms such as eye open or close, yawing, head tilting. Lane departure detection can strongly determine fatigue status along with these symptoms. These symptoms are making this research fruitfully. It is very difficult to detect the driver fatigue facial symptoms from the face image, because of variation on human face structure, diversity of face features, various pose, facial expression, brightness problem and shooting angle. The brightness problem is reduced by $\mathrm{YCbCr}$ conversion of RGB image. Large area of region is achieved through maximum connected area in binary image and other small area is rejected. Experimental result of the proposed method has been compared with other existing methods. Experimental result shows that the proposed method is more practical to implement in vehicle and less complex to detect the driver's fatigue symptoms.

\section{REFERENCES}

[1] N. Minoiu Enachea, M. Nettoa, S. Mammarb, B. Lusettia, "Driver steering assistance for lane departure avoidance", Control Engineering Practice, Volume 17, Issue 6, pp. 642-651, June. 2009.

[2] Saeid. Fazli, and Parisa. Esfehani," Tracking Eye State for Fatigue Detection", International Conference on Advances in Computer and Electrical Engineering, pp.17-18, Nov.2012.

[3] Arun Sahayadhas, Kenneth Sundaraj and Murugappan Murugappan, "Detecting Driver Drowsiness Based on Sensors: A Review “, ISSN 1424-8220, pp.16937-16953, Dec.2012.

[4] Paula Sind-Prunier, "Driver Safety through Human Factors Science and Practice ", the federation of behavioral", 314-6493.

[5] S. Ribarić, J. Lovrenčić, N. Pavešić, “A Neural-NetworkBased System for Monitoring Driver Fatigue",15th IEEE Mediterranean Electrotechnical Conference, pp. 13561361, Apr. 2010.

[6] Hua $\mathrm{Gu}$, Guangda Su, Cheng Du "Feature Points Extraction from Faces", November. 2003.
[7] M. H. Yang, N. Ahuja, "Detecting Faces in Images: A Survey", .in IEEE Transactionson Pattern Analysis and Machine Intelligence, Vol. 24, No.1, 2002.

[8] Miao, B. Yin, K. Wang, L. Shen, and X. Chen,"A Hierarchical Multiscale and Multiangle System for Human Face Detection in a Complex Background Using Gravity-Center Template," Pattern Recognition, vol. 32, no. 7, pp. 1237-1248, 1999.

[9] H. Rowley, S. Baluja, and T. Kanade, "Neural Network-Based ace Detection," Proc. IEEE Conf. Computer Vision and Pattern Recognition, pp. 20.

[10] M. Eriksson, and N.P. Papanikotopoulos, "Eye-tracking for detection of driver fatigue," in Proc .Int. Conf. Intelligent Transportation Systems, Boston, pp. 314-319, 1997.

[11] M. Young, Q. Wang, J. Yang, "Eye Location and Eye State Detection in Facial Images with Unconstrained Background", Journal of Information and Computing Science, Vol. 1, No. 5, pp. 284-289,2006.

[12] P. Bajaj, N. Narole, M. Sarada Devi, "Research on Driver's Fatigue Detection”, IEEESMC, Issue \#31, June. 2010.

[13] A Priya R. Lodha, Nitin R. Chopde," Analysis of Eye Fatigue Detection Method using Skin Color Modeling" International Journal of Advance Research in Computer Science and Management Studies, Volume 2, Issue 2, ISSN: 2321-7782, February. 2014.

[14] A. S. Md. Sohail and P. Bhattacharya, "Detection of Facial Feature Points Using Anthropometric Face Model", 2006.

[15] D Mandalapu Saradadevi, Dr. Preeti Bajaj," Driver Fatigue Detection Using Mouth and Yawning Analysis", International Journal of Computer Science and Network Security, VOL.8 No.6, June. 2008.

[16] J. Qiang, Z. Zhiwei, "Real time and non-intrusive driver fatigue monitoring", In: Proc. The 7th International IEEE Conference on Intelligent Transportation Systems, pp.657-662, 2004.

[17] Ayush Joshi, Shruti Gujrati, Amit Bhati," Eye State and Head Position Technique for Driver Drowsiness Detection", International Journal of Electronics and Computer Science Engineering, ISSN- 2277-1956, Volume 2, Number 3.

[18] K. C. Kluge, "Extracting road curvature and orientation from image edge points without perceptual grouping into features," Proceedings of the Intelligent Vehicles '94 Symposium, pp. 109-114, 1994.

[19] V. Gaikwad, S. Lokhande, "Real-Time Lane Departure Detection Based on Extended Edge-Linking Algorithm", 2nd International Conference on Computer Research and Development, pp. 725 - 730, 2010.

[20] Nicolas Gourier Daniela Hall James L. Crowley,” Facial Features Detection Robust to Pose, Illumination and Identity", pp. 617-622. IEEE, 2004.

[21] Y. Z. Jie Yang, Xufeng Ling and Z. Zheng, "A face detection and recognition system in color image series," Mathematics and Computers in Simulation, pp. 531-539, 2008. 
[22] Varsha Powar, Aditi Jahagirdar, Sumedha Sirsikar, “ Skin Detection in YCbCr Color Space", International Journal of Computer Applications, 2011.

[23] Fattah Alizadeh, Saeed Nalousi, Chiman Savari," Face Detection in Color Images using Color Features of Skin", World Academy of Science, Engineering and Technology, 2011.

[24] Aryuanto Soetedjo, Koichi Yamada, F. Yudi Limpraptono "lip detection based on normalized rgb chromaticity diagram", The 6th International Conference on Information \& Communication Technology and Systems, VI-63, ISSN 2085-1944.

\section{AUTHORS' PROFILE}

Md. Mehedi Hasan is currently pursuing B.Sc. degree program in Electronics and Communication Engineering in Khulna University of Engineering \& Technology, Khulna9203, Bangladesh. His research interest includes antenna design, machine learning, pattern recognition, neural networks, computer networking, VLSI system design, image processing, telecommunication and feature selection.

Md. Foisal Hossain is currently working as an Assistant Professor in Department of Electronics and Communication Engineering at Khulna University of Engineering \&
Technology, Khulna-9203, Bangladesh. He completed his B.Sc in Electrical and Electronic Engineering from Khulna University of Engineering \& Technology, Bangladesh. He completed his M.Sc and $\mathrm{PhD}$ in Information Engineering from Kyulcyas, Okinawa, Japan. His research interest includes Digital Image Processing, Digital Signal Processing, Medical Image Processing and Antenna Engineering.

Jag Mohan Thakur is currently pursuing B.Sc. degree program in Electronics and Communication Engineering in Khulna University of Engineering \& Technology, Khulna9203, Bangladesh. His research interest includes antenna design, machine learning, pattern recognition, neural networks, computer networking, VLSI system design, image processing, embedded system design, web security \& application, telecommunication and feature selection.

Prajoy Podder is currently pursuing B.Sc. degree program in in Electronics and Communication Engineering in Khulna University of Engineering \& Technology, Khulna-9203, Bangladesh. His research interest includes antenna design, machine learning, pattern recognition, neural networks, computer networking, VLSI system design, image processing, embedded system design, web security \& application, renewable power energy, biomedical, semiconductor devices, telecommunication and feature selection. 